\section{The challenges of maintaining non-COVID medicine services at Groote Schuur Hospital, Cape Town, South Africa, during COVID-19}

To the Editor: The article by Mendelson et al. ${ }^{[1]}$ describing the Herculean cross-discipline intervention to manage COVID-19 at Groote Schuur Hospital (GSH), Cape Town, South Africa, refers. An aspect of the response as yet not reported, although acknowledged, are the consequences of the COVID-19 response and its effect upon non-COVID general medical and routine public health services.

Public health services such as vaccination, tuberculosis diagnostics and HIV management services have been negatively affected. Factors such as the hard lockdown and individuals' own fears of COVID resulted in presentations to hospital services being delayed. Medicine services at GSH needed to continue despite the marked redistribution of staff to COVID-19 services. Inpatient general medicine services were scaled back to four wards, all geographically dislocated from the dedicated COVID-19 wards (for infection prevention and control (IPC) needs). Equally, patients with general medical presentations testing negative (person under investigation (PUI)) needed to be shifted to the non-COVID-19 service as expeditiously as possible to ensure a constant patient flow and alleviate bed pressures.

The vacuum created in the Department of Medicine by the redistribution of staff to COVID services created staffing challenges for the non-COVID-19 services. Colleagues from the divisions of Dermatology and Neurology were called upon and commendably supported our non-COVID-19 services. Unlike current policy, all patients were not automatically screened for COVID, unless regarded as a PUI. To run the non-COVID-19 medicine service, we adopted an 'all patients are COVID-positive until proven otherwise' approach. However, access to personal protective equipment (PPE) was different in that several PPE components, including N-95 masks, were understandably re-routed to the COVID service. Through a generous donation, we were able to access $\mathrm{KN}-95$ masks for medical and nursing staff, which were re-used for $1-2$ weeks at a time. This certainly assisted in elevating individuals' sense of personal protection, apart from all other standard routine measures.

Staffing in the non-COVID-19 medicine service remained challenging, as people acquired COVID-19 and had to quarantine and recover. In one instance in our service, 2 registrars and 4 interns all tested positive within a 48-hour period, leaving an entire firm almost without medical staff. In these instances, the true values of the department and individuals were witnessed in a sense of camaraderie and stepping up to get the job done with minimal complaints by registrars, medical officers, interns and consultants. Notably, several supernumerary registrars opted not to return to their home countries pre-lockdown and instead stayed and supported our effort. Equally, it would be remiss not to specifically acknowledge the dedication of our nurse colleagues who continued to deliver care under difficult circumstances where continued IPC practices were required and needed implementation and monitoring. An aspect that warrants specific comment is the venerable contribution of the intern cadre. Interns from across all disciplines were drafted into the COVID-19 service. This had to be done in a manner that did not disrupt the intern training programme, which has sub-minimum requirements in the various domains. A disruption to this programme would have had a marked and detrimental effect upon doctor development in the country. This was achieved to the absolute credit of our interns, who demonstrated the utmost commitment and professionalism. Throughout, several subspecialist service clinics were able to continue, albeit at a downscaled level and introducing protocols for clinics to run while mitigating COVID-19 risk. This was necessary because many subspecialist-run services manage patients who must have ongoing follow-up and access to their medicines. We hope that it has managed to prevent some of the adverse consequences on patient follow-up through COVID-19, but the closure of many other clinics has undoubtedly had an impact. This effect is evident by the surge in non-COVID admissions being experienced as COVID-19 admissions have steadily declined, a surge that is likely to continue for a time yet.

While teams were clearly important in managing COVID-19 at GSH, camaraderie was key to keeping the non-COVID-19 service functioning. Both were achieved, confirming the resilience and resourcefulness of staff in our Department and at the hospital in the face of adversity.

Mark Sonderup, Wendy Spearman, Ian Ross, Neliswa Gogela, Marc Combrinck, Mashiko Setshedi, Gregory Symons, Nicola Wearne, Shakeel Hoosain, Jean-Jacques Kruger, Bulelwa Mbena, Darlene Boayke, Tatenda Muchenje, Malcolm Masikati, Elisha Okeyo, Bolokang Makhura, Jean Botha, Lethabo Hlongwane, Ntobeko Ntusi

Department of Medicine, Faculty of Health Sciences, University of Cape Town and Groote Schuur Hospital, Cape Town, South Africa msonderup@samedical.co.za 1. Mendelson M, Booyens L, Boutall A, et al. The mechanics of setting up a COVID-19 response:
Experiences of the COVID-19 epidemic from Groote Schuur Hospital, Cape Town, South Africa. S Afr
Med J 2020;110(10):968-972. https:// doi.org/10.7196/SAMJ.2020.v110i10.15215

S Afr Med J 2020;110(12):1151. https://doi.org/10.7196/SAMJ.2020.v110i12.15392 